Revue des patrimoines

$28 \mid 2016$

Le moulage. Pratiques historiques et regards contemporains

\title{
Fernand Mayence et les moulages en Belgique : un musée universitaire (1927) et une reconstitution architecturale spectaculaire (1933)
}

Fernard Mayence and the plaster casts in Belgium: a university museum (1927) and a spectacular architectural reconstitution (1933)

\section{Bernard Van Den Driessche}

\section{(2) OpenEdition}

\section{Journals}

Édition électronique

URL : http://journals.openedition.org/insitu/12527

DOI : 10.4000/insitu. 12527

ISSN : 1630-7305

Éditeur

Ministère de la culture

Référence électronique

Bernard Van Den Driessche, «Fernand Mayence et les moulages en Belgique : un musée universitaire (1927) et une reconstitution architecturale spectaculaire (1933) », In Situ [En ligne], 28 | 2016, mis en ligne le 16 mars 2016, consulté le 19 avril 2019. URL : http://journals.openedition.org/insitu/12527 ; DOI : 10.4000/insitu. 12527

Ce document a été généré automatiquement le 19 avril 2019.

\section{c) (†)}

In Situ Revues des patrimoines est mis à disposition selon les termes de la licence Creative Commons Attribution - Pas d'Utilisation Commerciale - Pas de Modification 4.0 International. 


\section{Fernand Mayence et les moulages en Belgique : un musée universitaire (1927) et une reconstitution architecturale spectaculaire (1933)}

Fernard Mayence and the plaster casts in Belgium: a university museum (1927) and a spectacular architectural reconstitution (1933)

\section{Bernard Van Den Driessche}

Communication présentée lors des journées d'étude « Le moulage. Pratiques historiques et regards contemporains » organisées par la Cité de l'architecture et du patrimoine et le musée du quai Branly, les 14 et 15 novembre 2012.

1 La fin des années 1920 marque un tournant dans l'histoire des collections de moulages en Belgique dont l'apogée se situe comme ailleurs en Europe au XIXe siècle ${ }^{1}$. Notre pays y avait occupé une place de tout premier plan au niveau international, grâce aux actions menées par Henry Rousseau dont la carrière débute dans ce domaine en 1893 et s'achève en $1928^{2}$.

2 Dans ce contexte, il est opportun de s'arrêter sur deux grandes réalisations dues à Fernand Mayence (1879-1959). Professeur, conservateur, philologue et archéologue, il est une figure majeure de l'archéologie classique en Belgique ${ }^{3}$, attaché à l'université catholique de Louvain (UCL) à partir de 1907 et à la section des Antiquités des Musées royaux d'art et d'histoire de Bruxelles en 1913.

\section{Le musée d'archéologie classique de l'UCL}

Promu docteur en philosophie et lettres, groupe philologie classique à l'UCL en 1901, F. Mayence $^{4}$ séjourna en qualité de boursier du concours interuniversitaire dans les 
universités de Berlin, Halle et Paris en 1902 et 1903 avant d'être admis comme membre étranger de l'École française d'Athènes en 1904-1905. En 1907, il est appelé à enseigner les institutions de l'antiquité classique à l'UCL et dès 1908 il y joignit un cours d'archéologie classique.

Dans un bref article ${ }^{5}$, publié au début de sa carrière, il reconnaît le retard qu'accuse notre pays en ce qui concerne l'enseignement de l'archéologie et l'usage des moulages.

Cette double importance de l'archéologie, les Allemands l'ont parfaitement comprise, et il n'est plus aucune université d'Allemagne qui ne possède non seulement son cours d'archéologie, mais aussi son musée de plâtres.

Et d'ajouter :

Si l'enseignement de l'archéologie est représenté à Paris d'une façon brillante par des maîtres tels que les Collignon, les Pottier, les Reinach, il laisse encore cependant à désirer dans plus d'une université de France; mais comme le fait justement remarquer M. Perrot, le principe est posé, et, avec le temps, il sortira ses conséquences.

Pour conclure, il rappelle encore que

le gouvernement belge qui avait fait un premier pas dans la reconnaissance de l'importance des études d'archéologie classique en envoyant depuis 1900 deux délégués à l'École française d'Athènes, avait fait un pas décisif en instituant les grades de candidat, licenciés et docteur en art et archéologie.

7 Si l'histoire des collections de moulages à l'UCL débute dès $1864^{6}$, il faut attendre les premières " conférences d'archéologie classique ", inaugurées par F. Mayence, pour voir se constituer une collection de moulages antiques à usage exclusivement didactique. Dès l'année académique 1909-1910, le recteur de l'université accorde un petit crédit pour ces conférences ainsi que pour le musée et ses toutes premières acquisitions ${ }^{7}$. D'autres achats ont dû suivre, sans qu'on en connaisse le détail.

Mais c'est au lendemain de la Première Guerre mondiale que la collection fut réellement constituée. En effet, dans le cadre d'un des articles du traité de Versailles, l'office de la restauration de la bibliothèque de l'université de Louvain institua une commission mixte, belgo-allemande, pour présider au choix des objets à fournir par l'Allemagne dans le cadre des dommages de guerre et en particulier de l'incendie délibéré de la bibliothèque universitaire installée dans les halles, le bâtiment central de l'université. L'atelier de Berlin livra ainsi une série de moulages de l'Antiquité, du Moyen Âge et de la Renaissance ${ }^{8}$

9 Dans un élan de solidarité, des comités de soutien se créèrent également dans divers pays et rassemblèrent leurs efforts dans "L'œuvre internationale Louvain». Le comité national grec en particulier, suite aux contacts privilégiés qu'avait $\mathrm{F}$. Mayence avec son collègue $\mathrm{M}$. Svoronos, fit ainsi don d'une série de moulages des principaux chefs-d'œuvre de sculptures des musées d'antiquités pour un montant correspondant à 30000 drachmes de l'époque 9 . Les archives F. Mayence, qui ne conservent pas la liste de ces moulages, permettent de suivre du 30 mars 1920 au 21 août 1925, les diverses péripéties de l'envoi et de la réception d'un ensemble de 108 caisses pour un total de plus de $9000 \mathrm{~m}^{3}$ et 14 tonnes, acheminées par bateau d'Athènes à Anvers puis par train, du port belge à Louvain $^{10}$ !

10 L'université, via l'Office de la restauration de la bibliothèque et sur les instructions de $\mathrm{F}$. Mayence, passa également commande d'une soixantaine de moulages (dont la Victoire de 
Samothrace) à l'atelier des moulages de Bruxelles. La dépense totale pour cette acquisition en date du mois de juillet 1926 s'élevait alors à $22595 \mathrm{~F}$ belges ${ }^{11}$.

11 Les collections d'art et d'archéologie, réinstallées dans les halles universitaires, furent inaugurées en 1927, avec deux ans de retard, dans le cadre de la célébration du 500 anniversaire de l'UCL (1425-1925) ${ }^{12}$ (fig. 1, fig. 2). Toutefois, l'ouverture officielle du musée d'Archéologie classique eut réellement lieu le 22 mai 1930 à l'occasion d'une cérémonie d' " Hommage de reconnaissance de l'Université de Louvain à la Grèce ${ }^{13}$ ». La collection des moulages d'œuvres de l'Antiquité - Mésopotamie, Assyrie, Égypte, Mycènes, Crète, Grèce, Rome - atteignait alors le millier d'œuvres.

Figure 1

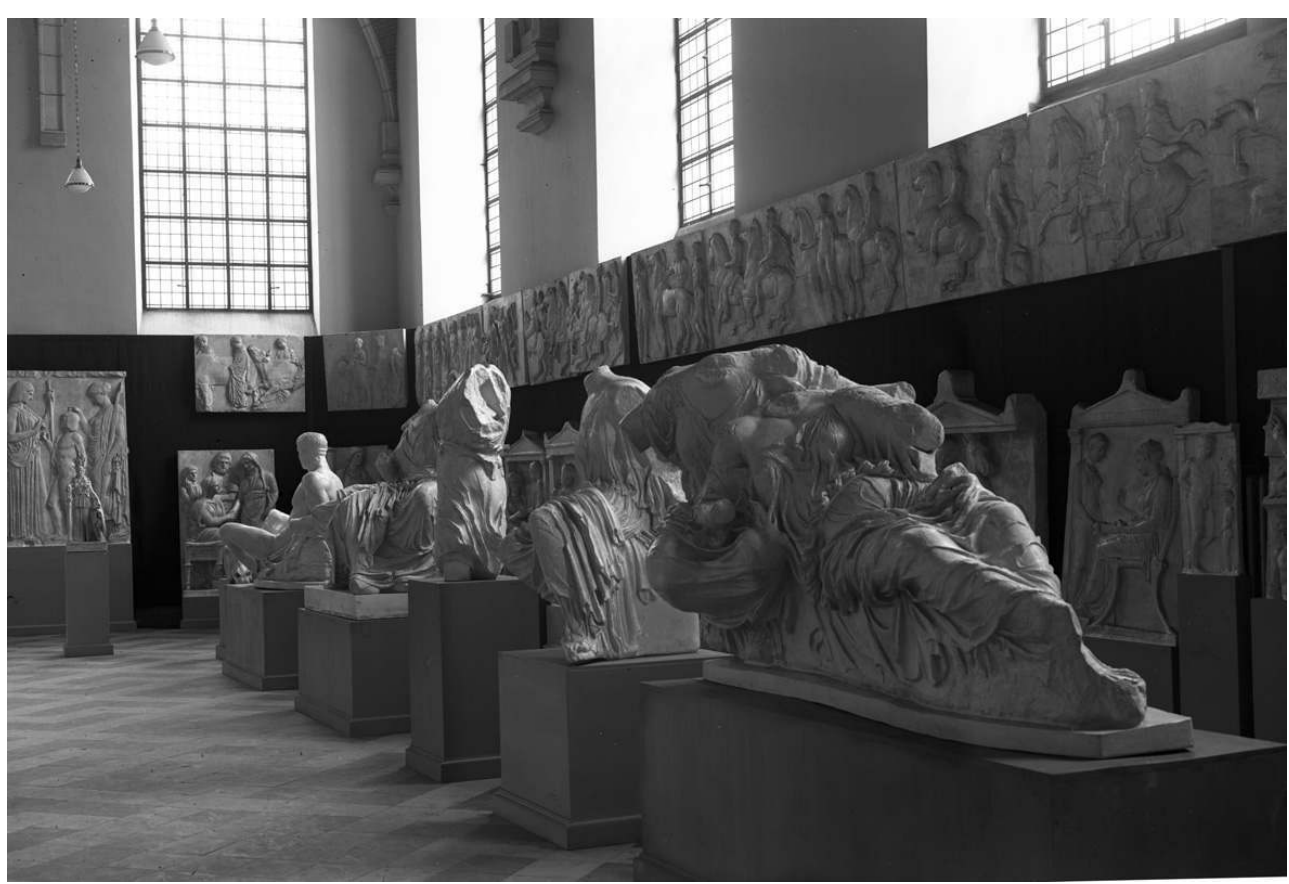

Le musée des Moulages de l'université catholique de Louvain en 1927.

(c) Archives F. Mayence. Musée de Louvain-la-Neuve. 
Figure 2

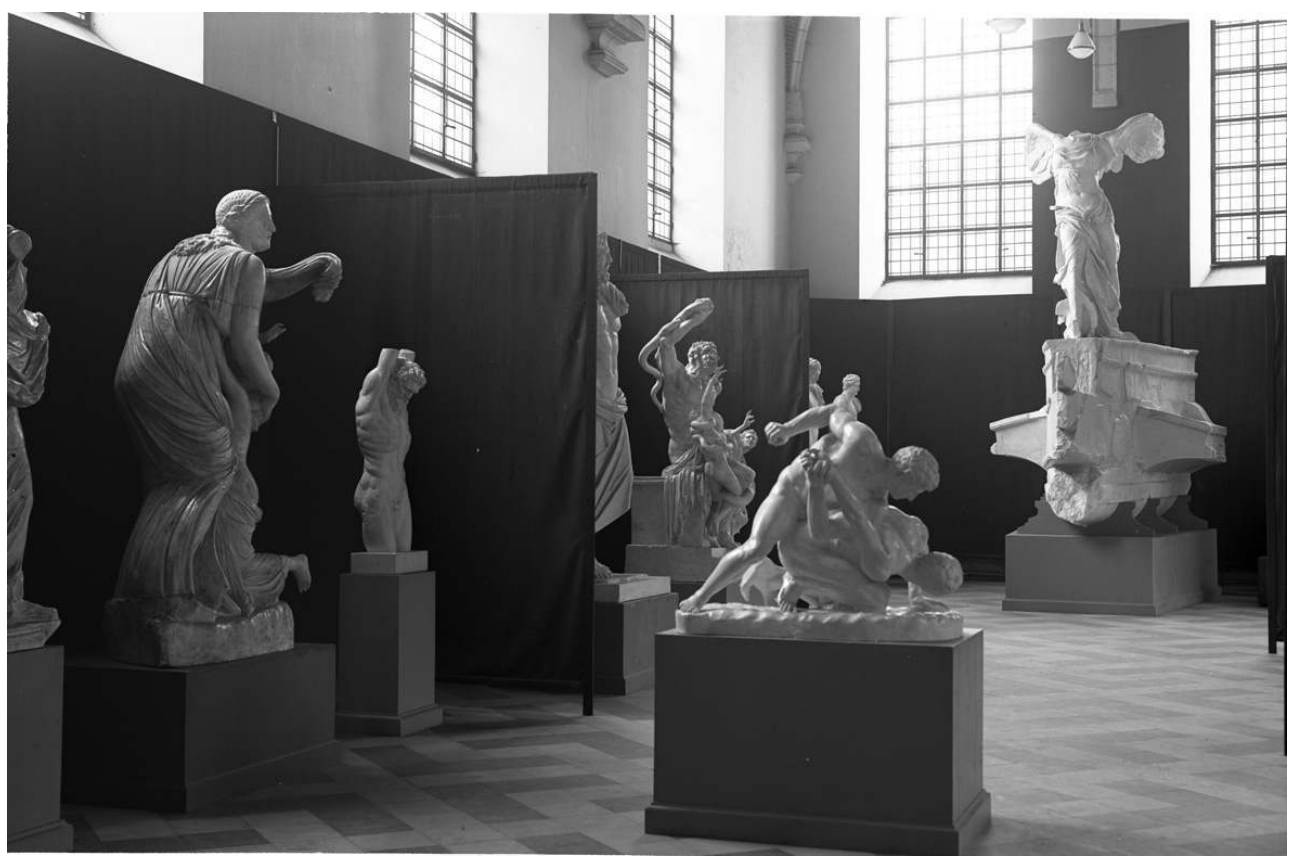

Le musée des Moulages de l'université catholique de Louvain en 1927.

(c) Archives F. Mayence. Musée de Louvain-la-Neuve.

12 Le choix des moulages constituant ce musée révèle une volonté, chez F. Mayence, de couvrir toutes les grandes périodes de l'Antiquité, mais aussi un maximum de formes et d'expression de l'art. Une dactyliothèque, des petits objets de fouilles (vases, bijoux, stucs, fragments de peintures, statuettes... sortis de l'atelier athénien de Gilliéron) côtoient les grands classiques de la sculpture en ronde-bosse ou en bas-relief, mais aussi des éléments d'architecture (chapiteaux, bases de colonne, fragments d'entablement), y compris par exemple un siège du théâtre de Dionysos et une rare maquette de ce même théâtre sortie de l'atelier G. Walger à Berlin. L'orientation essentiellement pédagogique liée au contenu de ses cours d'archéologie est manifestement voulue et affirmée. Une note personnelle consignée lors d'une visite de la collection des moulages de Lyon entre 1928 et 1930 le confirme :

On voudra bien se rappeler que cette collection dépend d'un des enseignements de l'Université et n'a été créée que pour l'usage même de cet enseignement : elle n'est pas du tout un musée public. Les facilités accordées aux visiteurs, plus grandes et plus libérales que nulle autre part, y resteraient nécessairement subordonnées aux besoins du service; et les consignes données à ce sujet par le professeur responsable y doivent être respectées de chacun ${ }^{14}$.

Malgré de très importants dommages subis encore durant la Seconde Guerre mondiale, la collection des moulages restera utilisée, mais de moins en moins, pour l'enseignement de l'histoire de l'art et de l'archéologie jusqu'à la réorganisation complète du bâtiment pour les services administratifs de l'université en 1960. Elle comptait alors encore quelque 600 pièces. 
Figure 3

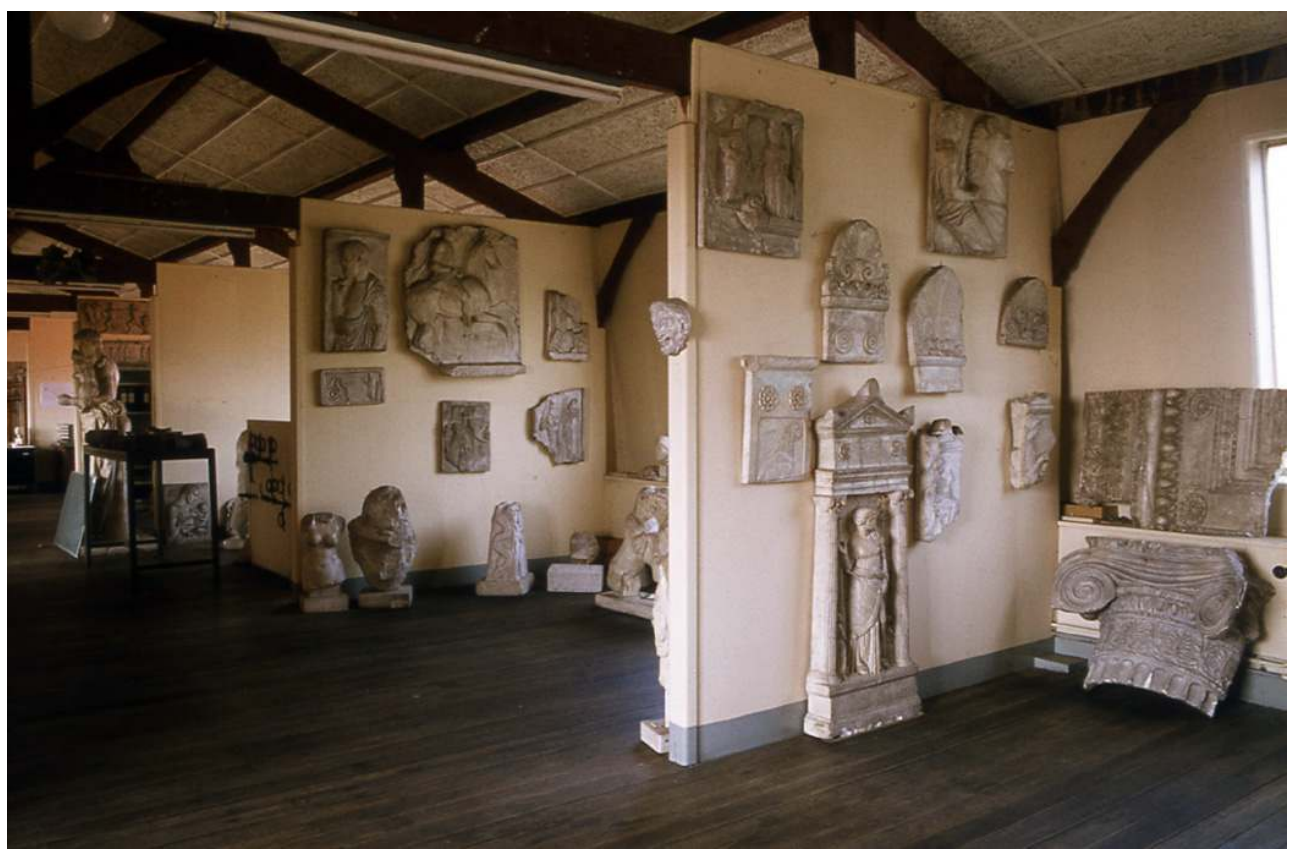

Une partie des collections des moulages de l'Antiquité classique au $3^{e}$ étage de l'Institut supérieur d'archéologie à Louvain. Situation en 1974.

Phot. Van den Driessche, Bernard. (c) Archives du Musée de Louvain-la-Neuve.

14 Rassemblés dans le bâtiment de l'Institut d'archéologie (fig. 3) puis soumis à la loi du partage entre les deux universités (UCL-KUL) ${ }^{15}$, les moulages attribués à l'UCL seront transférés en 1979 (fig. 4) sur le nouveau site de l'université à Louvain-la-Neuve. Ils ont été alors répartis entre une réserve (fig. 5), des salles et couloirs des nouvelles halles universitaires et de la bibliothèque générale des sciences humaines. Ces moulages, gérés avec l'ensemble du patrimoine du musée de Louvain-la-Neuve, participent occasionnellement au concept du musée du dialogue lors d'expositions temporaires et font encore l'objet d'exercices pratiques pour les étudiants de la section archéologie de l'Antiquité. Un projet de présentation de quelques moulages représentatifs, parmi les collections d'originaux, est en préparation pour le bâtiment de la bibliothèque des sciences exactes qui deviendra prochainement le nouveau musée de l'Université. 
Figure 4
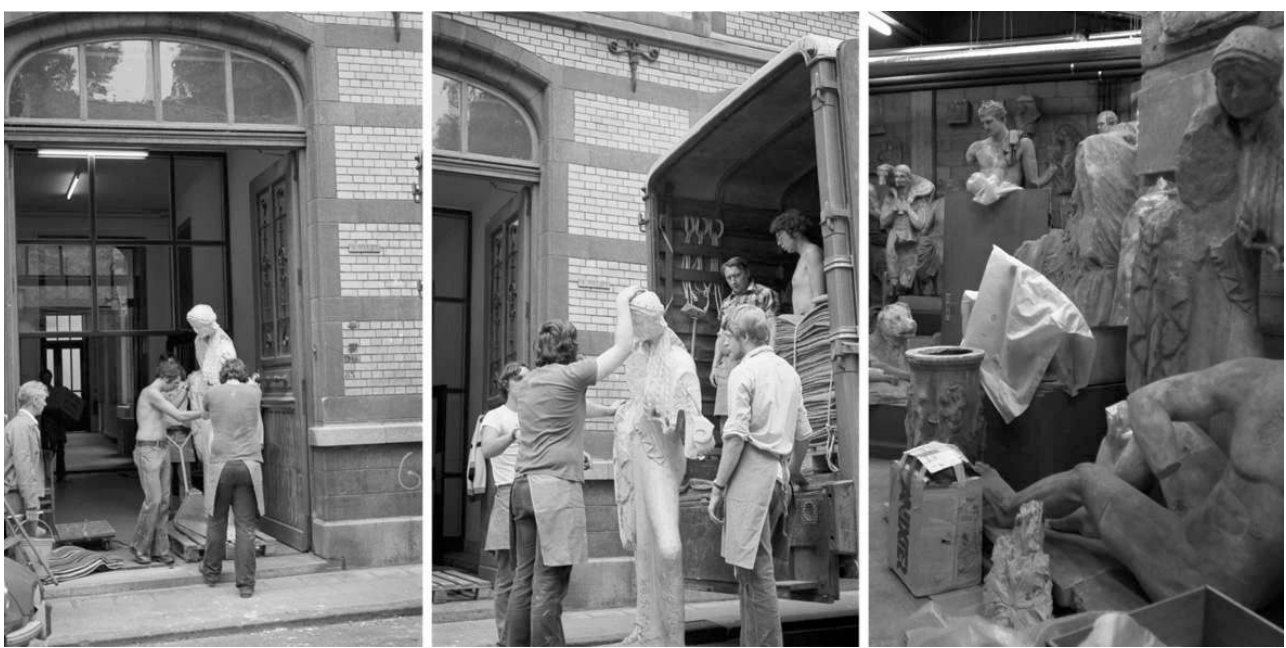

Transfert des moulages de Louvain (Leuven) à Louvain-la-Neuve (1979).

Phot. Van den Driessche, Bernard, Piron, Jacques. (c) Archives du Musée de Louvain-la-Neuve

Figure 5

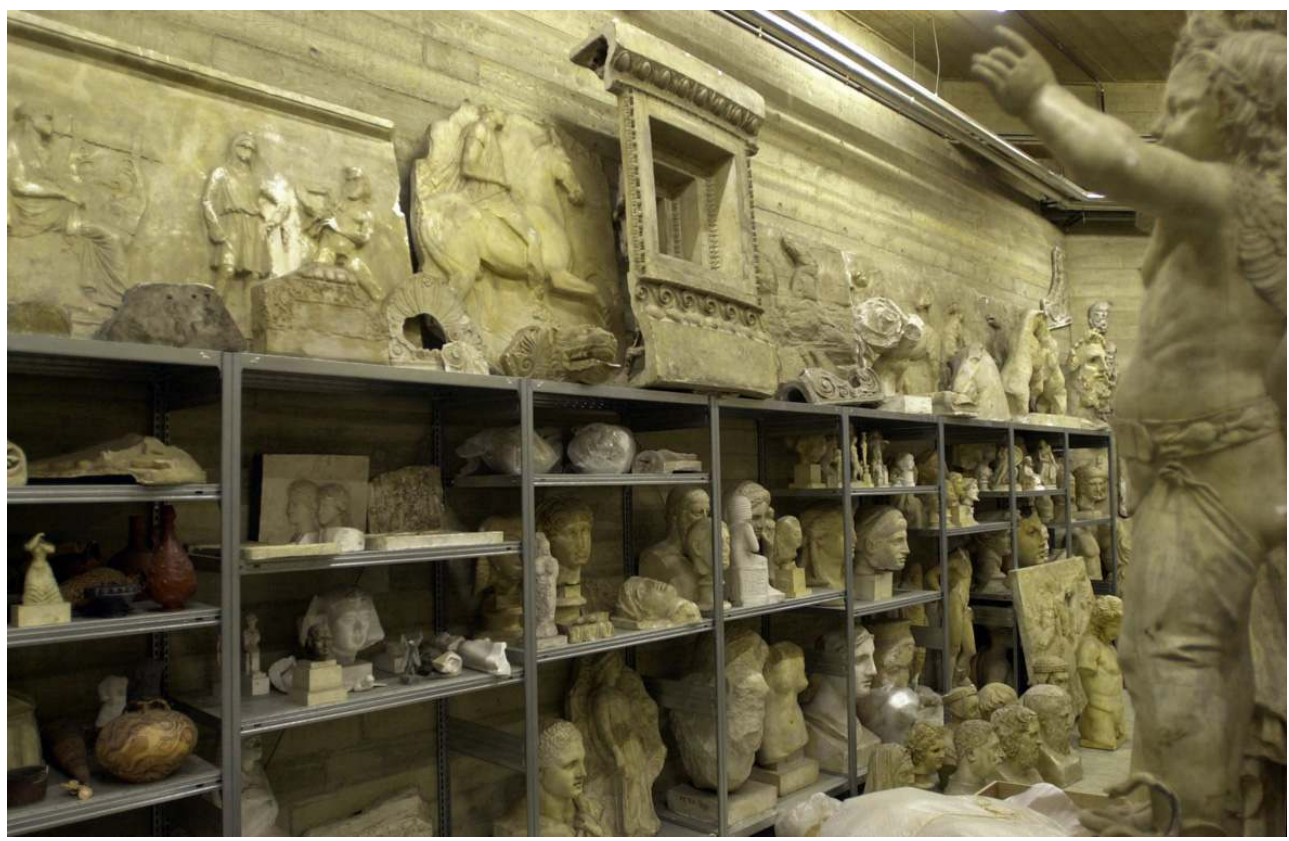

Vue partielle de la réserve des moulages à Louvain-la-Neuve (1999)

Phot. Van den Driessche, Bernard. (c) Archives du Musée de Louvain-la-Neuve.

\section{Reconstitution de la colonnade d'Apamée}

Peu après l'inauguration du musée universitaire, l'année 1928 devait marquer un nouveau développement de l'activité scientifique de F. Mayence. Ayant obtenu pour la Belgique une concession de fouilles en Syrie, à Apamée sur l'Oronte, Franz Cumont désigna F. Mayence comme directeur des fouilles, en collaboration avec l'architecte Henry Lacoste ${ }^{16}$. Celui-ci, comme l'archéologue, avait été membre de l'École française d'Athènes. 
16 Après une mission exploratoire en 1928, en vue de déterminer les possibilités et les conditions de cette entreprise, F. Mayence y dirigea six campagnes de fouilles de 1930 à 1937. Alors que le rapport des fouilles de la première mission de 1930 n'est pas encore rédigé, $\mathrm{F}$. Mayence s'apprête à mettre sur pied la deuxième campagne qui durera du 15 octobre au 6 décembre 1931. L'architecte H. Lacoste l'accompagnera et, pour la première fois, dans un courrier du 27 août 1931, il est noté que : « la mission s'adjoindra les services d'un mouleur qui a travaillé à Leptis Magna et qui fournira une aide précieuse pour les reconstitutions $»^{17}$. Il s'agit d'Alberto Scanzani à propos duquel nous ne possédons pas d'autres informations à ce jour, sinon qu'il est l'auteur d'une maquette en plâtre, à échelle réduite, de la colonnade d'Apamée exécutée en deux exemplaires ${ }^{18}$.

La correspondance des deux premières campagnes de fouilles, conservée aux archives du musée de Louvain-la-Neuve, est riche de détails sur les difficultés quotidiennes d'approvisionnement, du transport, de la qualité variable du plâtre, de la prise de moulages menée sur le site, des dépenses occasionnées par ce travail et enfin de l'acheminement des moules et moulages jusqu'à Beyrouth, port d'embarquement vers la Belgique. C'est parfois au jour le jour que le directeur des fouilles nous en informe dans ses notes personnelles ou dans la correspondance adressée à Franz Cumont et plus encore dans celle envoyée à son épouse ${ }^{19}$. Ces courriers sont également accompagnés des premières photographies des tambours de colonnes moulés (fig. 6) ; elles constituent un témoignage exceptionnel de cette entreprise, en sachant qu'à son terme, elle rassembla $24 \mathrm{~m}^{3}$ de moulages en 1932 outre les 18 caisses déjà envoyées dès $1931^{20}$. F. Mayence pouvait réellement affirmer avec toute la modestie qui était la sienne : «Quand on verra dans les salles du Musée de Bruxelles les moulages des monuments d'Apamée, on ne se doutera pas de toute la peine qu'ils auront coûtés $»^{21}$.

Figure 6
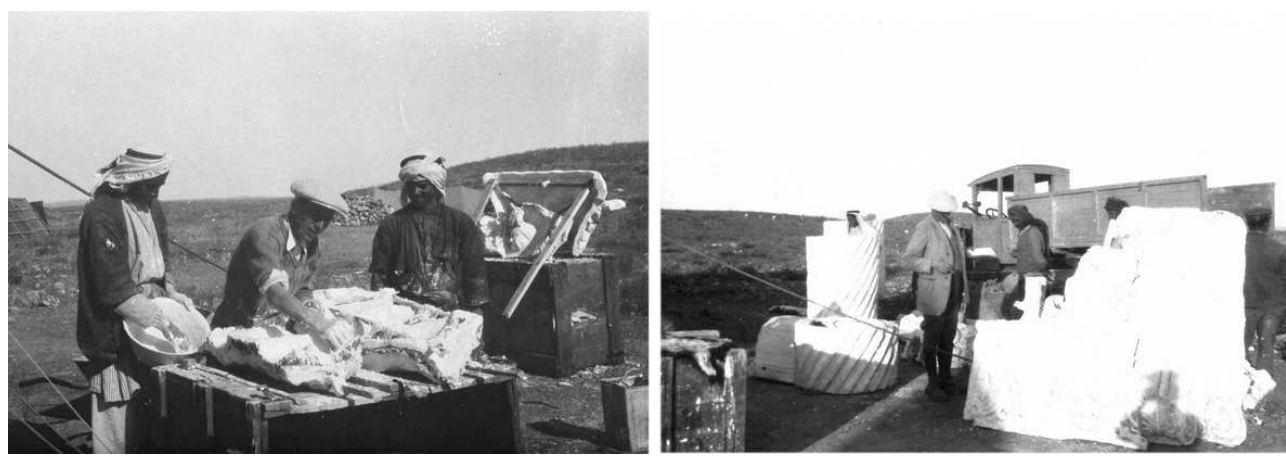

Apamée sur l'Oronte, 1932. A. Scanzani au travail et F. Mayence inspectant des moulages avant leur transport.

(C) Archives F. Mayence. Musée de Louvain-la-Neuve.

Au musée même, l'entreprise fut menée rondement grâce au travail d'Henry Lacoste qui en avait dressé les plans et suivi l'exécution. Important fut également l'investissement de l'atelier des moulages des Musées royaux d'art et d'histoire sous la houlette de son mouleur M. Piroux, Jean Troupin en étant le responsable. Cet ambitieux projet avait été accueilli avec enthousiasme par le conservateur du musée de l'époque, Jean Capart, qui affecta une partie du hall de l'Antiquité (l'aile Sud) dont le grand vaisseau « allait pour la première fois abriter une pièce en rapport avec ses vastes proportions ". 
Le 18 mars 1933, soit deux ans à peine après les premières prises d'empreintes sur le terrain, la salle d'Apamée est inaugurée très solennellement en présence de S.M. le roi Albert $\mathrm{I}^{\mathrm{er}}$, de la reine Élisabeth et d'un parterre d'autorités politiques, académiques et scientifiques (fig. 7).

Figure 7

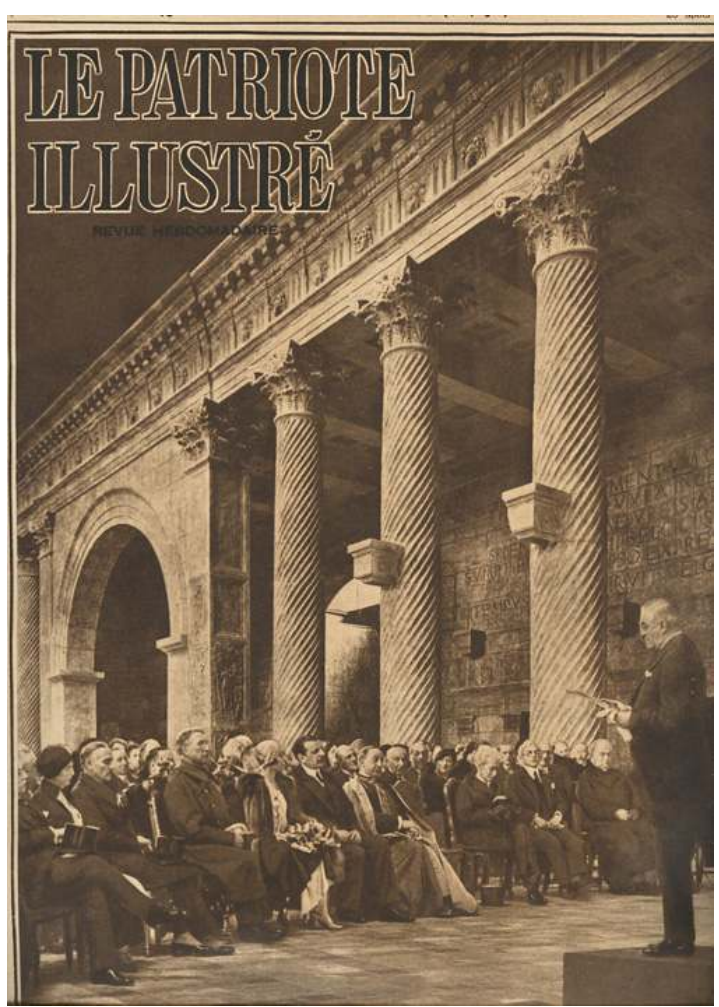

Couverture de l'hebdomadaire Le patriote illustré du 26 mars 1933. Archives F. Mayence. Musée de Louvain-la-Neuve.

(c) Le patriote illustré.

Cette "reconstitution loyale et sincère ${ }^{22}$ " se veut à l'époque un modèle de rigueur associant méthode scientifique et vocation didactique «jusqu'à la teinte de la patine appliquée sur le plâtre, qui évoque avec bonheur les reflets rougeâtres du calcaire d'Apamée ». Le caractère exceptionnel de cette réalisation repose également sur le fait que l'anastylose d'une partie de la colonnade de l'axe principal de la cité, soit quelque $1600 \mathrm{~m}$ de long sur 23,5 m de large, ne sera mise en œuvre partiellement que dans les années 1960. C'est une partie d'un des portiques qui s'élevait des deux côtés de la voie principale que présente la reconstitution. À une série de 7 colonnes succède en façade un grand arc, accosté de deux pilastres, qui surmonte le débouché dans l'artère principale d'une voie secondaire. Au-delà de l'arc, une huitième colonne annonce la continuation du portique. Les 8 colonnes, hautes de $6,75 \mathrm{~m}$, d'un diamètre de $90 \mathrm{~cm}$ et distantes de $3 \mathrm{~m}$ d'axe en axe, exécutées au départ du même exemplaire reproduit huit fois, sont celles du type torse et à arêtes vives. Elles ont été remontées par erreur dans le même sens, alors qu'il s'est avéré par la suite qu'il y avait un jeu subtil d'alternance du sens des torsades. Tous les détails de l'entablement ont été également moulés sur place. Des copies exactes des différents éléments qui le composaient, architrave, frise, corniche, ont été prises sur une longueur de deux à trois mètres; ces copies ont été répétées et juxtaposées pour 
former l'entablement du portique reconstitué. Enfin, le mur du fond du portique est la reproduction fidèle de celui qui a été découvert sur une hauteur de 3 à 6 mètres. Dans cette reconstitution, seul le plafond, reproduisant un modèle à caisson, était hypothétique en l'absence de tout vestige conservé.

Un plan et une coupe de la charpente en bois de cet ensemble révèlent le besoin de $23 \mathrm{~m}^{3}$ de boiserie pour un total de 20000 FRB de l'époque. La reconstitution, accompagnée de documents photographiques, de fragments architectoniques originaux et de mosaïques, occupe alors les $35 \mathrm{~m}$ de la largeur du fond de la salle sur une hauteur de 12 mètres.

La même salle abrite également, dans une réorganisation plus large de cette zone du musée, une partie des collections d'archéologie de l'Antiquité classique réaménagée également par F. Mayence qui en était le conservateur. En outre, une cinquantaine de moulages de sculptures classiques épargnés lors de la suppression du musée des moulages y étaient exposés à proximité ${ }^{23}$.

24 Cependant, le soir du 19 février 1946, le feu prend dans une tour d'angle du grand hall du Cinquantenaire et se propage rapidement dans une partie du bâtiment ${ }^{24}$. Le portique est entièrement détruit (fig. 8) ainsi que d'autres objets exposés et $F$. Mayence se voit à nouveau touché personnellement par la perte de nombreux documents de fouilles déposés dans son bureau, et dont une grande part avait déjà été détruite lors du bombardement et de l'incendie d'une aile des halles de Louvain à peine deux ans auparavant. Ce double drame devait affecter profondément le caractère de cet homme qui avait mis tant d'énergie dans sa double carrière professionnelle et la réalisation des deux projets évoqués ici.

Figure 8

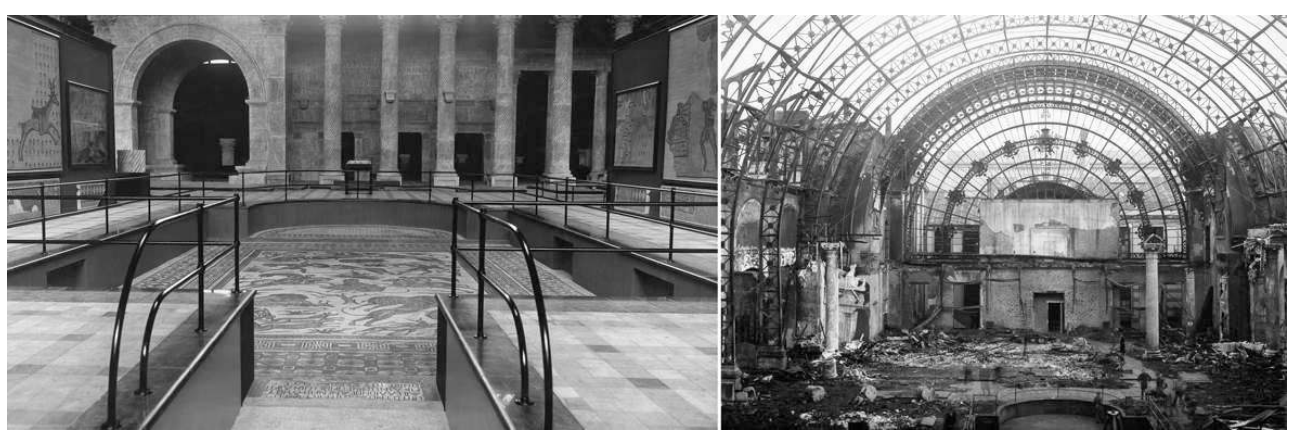

La salle d'Apamée aux Musées royaux d'art et d'histoire : état avant et après l'incendie de 1946. Nég. b2002 et m681.

(c) KIK/IRPA.

Henri Lavachery, le nouveau conservateur en chef du musée depuis 1942, est alors favorable à la reconstruction du bâtiment selon des plans de H. Lacoste. Il soulignait que cette aile devait certainement abriter la salle de Rome - avec la maquette de Pol Bigot acquise et installée au musée en 1950 grâce à l'action de Lacoste et celle de ses élèves de l'Académie royale des beaux-arts - et la salle d'Apamée.

En 1953, tout en reconnaissant les lacunes et erreurs du portique de 1933, Lacoste dessine un avant-projet de bâtiment qui s'organise entièrement autour de la mise en valeur de la grande colonnade d'Apamée. Il concevait alors de reconstituer, sur un premier étage et sur toute la longueur du hall cette fois, les deux côtés de la voie principale avec leurs 
colonnes, et au niveau des grandes arcades marquant le croisement avec le cardo de la ville, il imaginait encore de dresser, comme sur le site, la reproduction d'une des colonnes votives à base concave. Dans le même vaste hall reconstruit, il plaçait dans le fond le moulage du fronton oriental du Parthénon, ainsi qu'autour de la grande mosaïque de la chasse découverte à Apamée, divers moulages, tels la tribune complète des caryatides, la colonne des Naxiens à Delphes, etc. appartenant à l'ancien musée des moulages. Tout cela, il le voulait, comme F. Mayence, pour donner à ce lieu une vocation didactique, où les reconstitutions, les moulages, les relevés et les photographies sont appelés à jouer un rôle grandissant, si l'on défend, comme ils le faisaient tous deux, le principe que les vestiges archéologiques doivent rester in situ. Cet ensemble ne fut cependant pas réalisé comme tel.

Finalement, l'aménagement complet de la nouvelle aile ( $80 \mathrm{~m}$ de long sur $50 \mathrm{~m}$ de large et $25 \mathrm{~m}$ de haut) réservée aux collections de l'Antiquité sera confié aux architectes Robert Puttemans ${ }^{25}$ et Charles Malcause, les travaux s'échelonnant de 1956 à 1966 (fig. 9). F. Mayence, décédé en 1959, ne pourra cependant pas en apprécier le résultat. Quant à $\mathrm{H}$. Lacoste et son collaborateur Paul Mignot, ils dirigent uniquement la reconstitution du portique de 1933, érigé dans l'axe longitudinal sur une galerie du premier étage, à partir des creux heureusement conservés par l'atelier des moulages du musée.

Figure 9

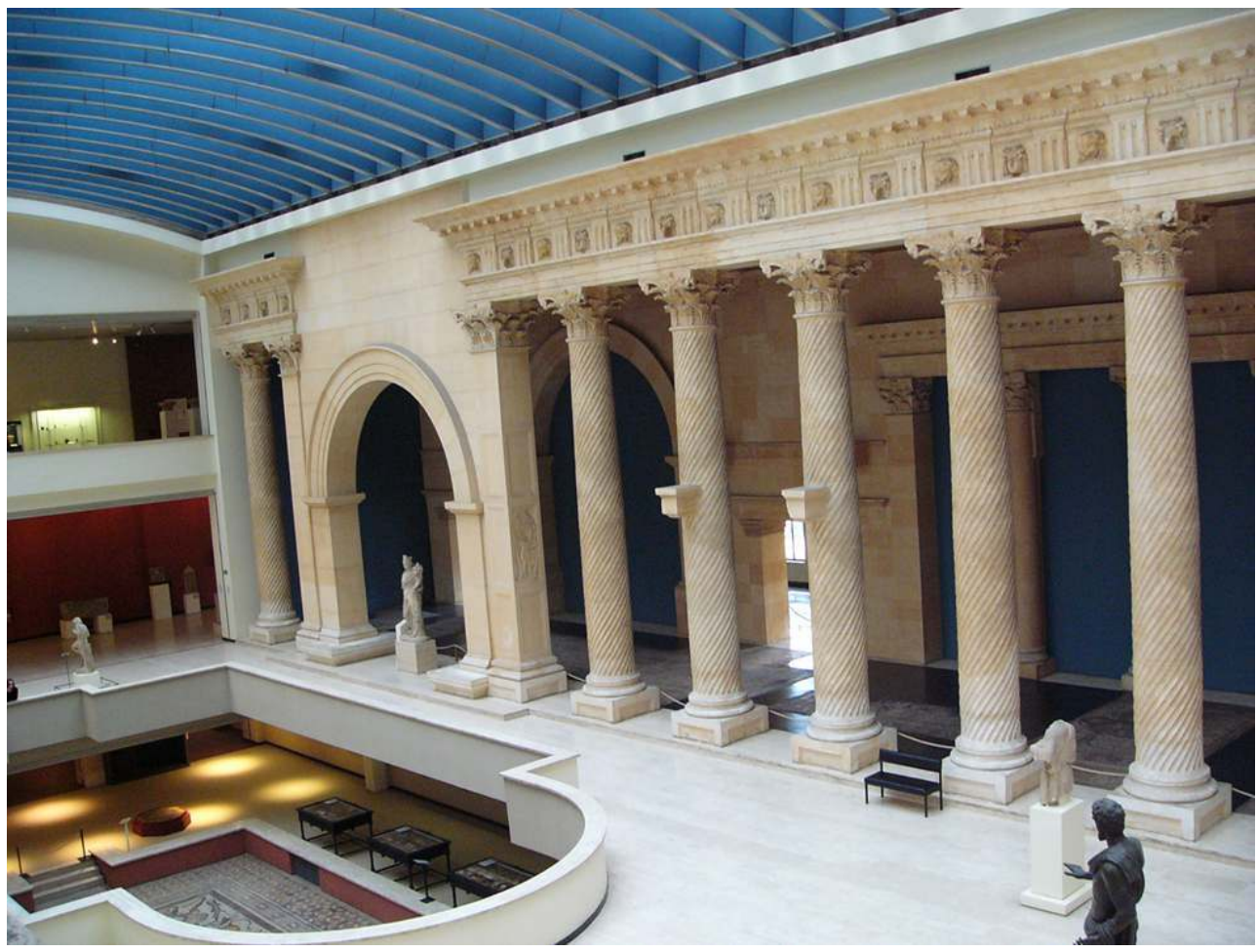

La salle d'Apamée dans son état actuel.

Phot. Van den Driessche, Bernard, 1999. (c) Bernard Van den Driessche.

L'espace ainsi reconstruit offre depuis son achèvement la sensation de grandeur liée au style impérial du site d'Apamée et, par la mise en place de la nouvelle verrière, la qualité de la lumière naturelle est particulièrement appréciée, même si elle n'égale pas celle du ciel syrien. 
La mise en œuvre du "musée d'Archéologie classique» à l'UCL s'inscrit dans une tradition attestée au sein des universités européennes à partir du XVIII ${ }^{e}$ siècle

F. Mayence veut ainsi offrir à son université un outil au service de l'enseignement de l'archéologie classique digne des modèles allemands, anglais, français, italiens qu'il connaissait pour en avoir fréquenté certains au tout début de sa carrière. Cette démarche s'inscrivait également dans une volonté du recteur de l'époque, Mgr Paulin Ladeuze, de doter l'université d'outils pédagogiques dans plusieurs facultés, faute de pouvoir reconstituer le précieux patrimoine des manuscrits anciens et œuvres originales détruits par l'incendie criminel de la bibliothèque en 1918.

1 Le hasard de l'histoire a voulu que cette entreprise ait débuté à l'époque où le « musée des Moulages » de Bruxelles fermait ses portes avec le départ à la retraite d'Henry Rousseau. La scission de l'université catholique de Louvain a cependant réduit la visibilité de cette réalisation, la collection ayant été partagée entre deux institutions distinctes et géographiquement séparées, sans qu'un espace muséal de qualité n'ait jamais été réalisé dans aucune des deux universités.

Si la maquette de la colonnade d'Apamée (fig. 10) réalisée en deux exemplaires à une date incertaine relève bien du concept d'objet d'exposition, elle constitue, comme cela a été écrit par ailleurs, un support d'enseignement, car elle analyse, par l'écorché et la partie, un aspect de l'édifice, qu'il soit structurel, ornemental ou distributif. Divers colloques ou publications ont mis l'accent sur les fonctions multiples des maquettes à échelle réduite, leur histoire, leurs techniques de réalisation et leurs limites de représentation « dans la mesure où la réalité qu'elle représente est excessivement réduite et attachée surtout aux volumes et non aux espaces ${ }^{26}$. Avec la 3D, la maquette numérique propose actuellement de redonner cette notion d'espace ${ }^{27}$.

Figure 10

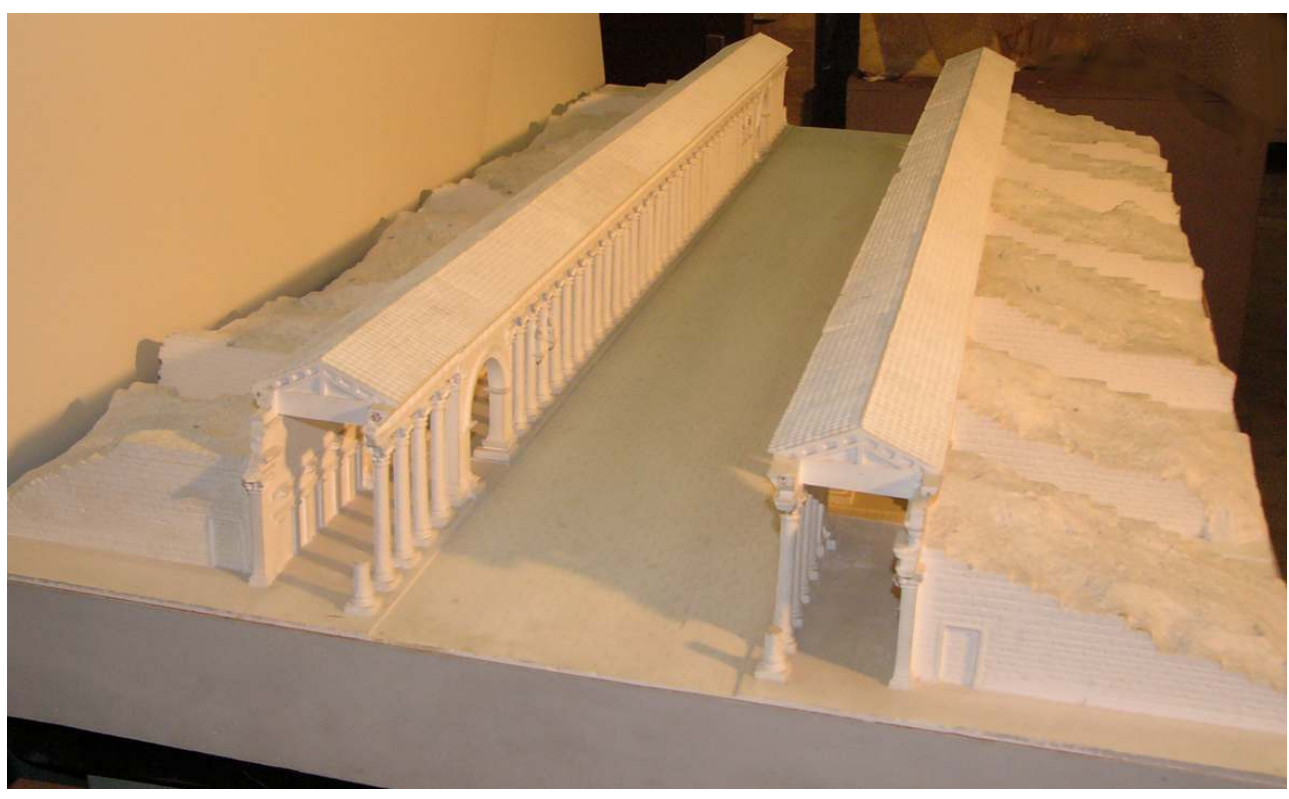

La maquette d'Apamée réalisée par A. Scanzani dans les collections du musée de Louvain-la-Neuve. Phot. Van den Driessche, Bernard, 2010. (c) Bernard Van den Driessche.

Mais la relation physique entre le corps et l'espace ne reste possible qu'avec la reconstitution à échelle réelle. C'est ce qu'ont voulu F. Mayence et $\mathrm{H}$. Lacoste à Bruxelles, 
à la suite des exemples des musées de moulages d'architecture du XIX ${ }^{e}$ siècle et en particulier celui des Monuments français qu'ils appréciaient tous deux ${ }^{28}$. Et ce n'est pas pour rien que la reconstitution du portique d'Apamée qui constituait un passage « du domaine scientifique au domaine de l'éducation populaire » a connu un réel succès. Sa réalisation a été répercutée lors de son inauguration par des dizaines d'articles de presse très élogieux et a contribué en outre à prolonger l'octroi de financement pour la poursuite des campagnes de fouilles dans un contexte économique difficile. Aujourd'hui encore, sa présence au sein des Musées royaux d'art et d'histoire de Bruxelles reste le point d'orgue d'une visite de la section consacrée à l'Antiquité classique et plus particulièrement à l'Empire romain. C'est à Fernand Mayence et à Henry Lacoste que le musée doit cette réussite et il est à regretter que cela ne soit pas signalé visiblement au public.

\section{Biographie}

Licencié en histoire ancienne et en archéologie de l'université catholique de Louvain. Boursier des accords culturels belgo italien, bulgare et ex-URSS. Participation aux missions archéologiques belges en Italie (Alba Fucens et Ordona) et en Syrie (Tell Kannas). Cofondateur du Musée de Louvain-la-Neuve et successivement conservateur-adjoint, administrateur et archiviste (1979-2010). Cofondateur et ancien président de l'association Musées et Société en Wallonie (regroupant actuellement plus de 160 institutions muséales). Membre fondateur du Comité international des musées et collections universitaires (UMAC) au sein de l'ICOM. Administrateur de l'Association internationale pour la conservation et la promotion du moulage et webmestre de son site internet "Plastercastcollection.org ». Articles publiés dans le domaine du dessin archéologique, de la bijouterie grecque hellénistique, de la muséologie et des moulages.

\section{NOTES}

1. - Pour les moulages dans les académies dans les anciens Pays-Bas méridionaux: LOIR, Christophe. «La Belgique et la perception de l'art antique avant 1830 : le développement des collections de plâtres de statues antiques à usage pédagogique ». Dans TSINGARIDA, Athéna et VERBANCK-PIERARD, Annie. Appropriating Antiquity - Saisir l'Antique. Collections et collectionneurs d'antiques en Belgique et en Grande-Bretagne au XIX siècle. Bruxelles, 2002, p. 33-72. Pour une approche de l'histoire des moulages en Belgique : VAN DEN DRIESSCHE, Bernard. «Les moulages en plâtre dans les académies et les universités belges ». La vie des musées, 2000-2001, 15, p. 62-66. VAN DEN DRIESSCHE, Bernard. «Le jardin des plâtres ». Courrier du passant. Bulletin bimestriel du Musée de Louvain-la-Neuve, 2001, 68, avril-mai, p. 2-28. VAN DEN DRIESSCHE, Bernard. "L'Antiquité et les moulages en plâtre en Belgique : 1830-1930». Dans TSINGARIDA, Athéna et VERBANCK-PIERARD, Annie. L'Antiquité au service de la modernité? La réception de l'Antiquité classique en Belgique au XIX ${ }^{e}$ siècle. (Actes du colloque international, Bruxelles 27-29 avril 2005). Bruxelles, 2008, p. 341-353.

2. - Il avait été conservateur de la «section d'Art monumental des Musées royaux des arts décoratifs et industriels " qui rassemblait en un seul lieu l'ancien musée des Plâtres et le musée des Échanges sur le site des arcades du Cinquantenaire à Bruxelles. La dernière édition de son catalogue (1926) dénombrait 5135 numéros pour l'ensemble des sections. Dès 1885 et en 1927, il 
est très impliqué dans ce qui devient le "Programme de l'office international des musées ». Mouseion, 1927, I (avril), p. 11-18. « La coopération des musées de moulages ». Mouseion, 1927, I (avril), p. 22-27 et «La coopération des musées de moulages. Accord entre musées et ateliers de moulages ». Mouseion, 1928, 4 (avril), p. 43-70. Rappelons enfin, en 1929, le catalogue Exposition internationale de moulages. Athènes, Berlin, Bruxelles, Florence, Londres, Paris (Société des Nations. Office international des musées de l'institut international de coopération intellectuelle). Paris et Bruxelles: (Éd. G. Van Oest), 1929. «Exposition de moulages». Mouseion, 1929 (décembre), 9, p. 304 et 1931, 13/14, p. 134-135. Pour tout ceci, voir également MONTENS, Valérie. Les moulages des Musées royaux d'art et d'histoire. Histoire de la collection et de l'atelier des reproductions en plâtre. Bruxelles: MRHA, 2008, p. 39-51, et sa contribution dans la présente publication. Voir, dans ce numéro l'article de Valérie Montens : La création d'une collection nationale de moulages en Belgique : http://insitu.revues.org/12564.

3. - Son nom n'a pourtant pas été retenu dans l'ouvrage de GRAN-AYMERICH, Ève. Les chercheurs du passé 1798-1945. Aux sources de l'archéologie. Paris : CNRS, 2001.

4. - DE RUYT, Franz. «Fernand Mayence. Professeur émérite de la Faculté de Philosophie et Lettres. 1879-1959». Annuaire de l'Université catholique de Louvain, 1957-59, III, p. 240-251. DE RUYT, Franz. « Notice sur Fernand Mayence ». Annuaire de l'Académie Royale de Belgique (Classe des Beaux-Arts), 1963, CXXIX, p. 177-190. VERHOOGEN, Violette. «Fernand Mayence». Biographie nationale, 1976, XXXIX, fasc. 2, p. 677-683. [VAN DEN DRIESSCHE, Bernard]. « Entre Grèce et Syrie. Fernand Mayence (1879-1959) ». Courrier du passant. Bulletin bimestriel du Musée de Louvain-la-Neuve , 1999, 60, p. 2-20.

5. - MAYENCE, Fernand. "L’importance des études archéologiques». Annuaire du Cercle pédagogique des professeurs de l'enseignement moyen sortis de l'Université de Louvain, $2^{\mathrm{e}}$ année, 1903-1904, fasc. 3, p. 11-15.

6. - VAN DEN DRIESSCHE, Bernard. «D'une collection de moulages à un musée universitaire public ». Dans LLINAS, Christian (éd.). Moulages. Actes des rencontres internationales sur les moulages, 14-17 février 1997, Montpellier, 1999, p. 109-117.

7. - MAYENCE, Fernand. «Conférences d'antiquité classique ». Annuaire de l'Université catholique de Louvain, 1910, p. 520-521. Il s'agissait de : la stèle funéraire d'Hégéso, la tête de la Pallas de Velletri, la tête de Zeus Otricoli, deux plaques de la frise du Parthénon, la statue d'Artemis Laphria, un buste d'Homère, deux têtes archaïques (du musée de Bruxelles), les têtes de la Vénus de Milo, du Laocoon, de Dionysos et un bas-relief archaïsant (du musée de Dresde).

8. - Les professeurs R. Lemaire, R. Maere, Éd. Remy et F. Mayence ont alors élaboré un plan pour la constitution d'un musée des moulages d'œuvres conservées dans les principaux musées d'Europe. Voir note 1.

9. - Ceuvre internationale Louvain. Bulletin publié par le Commissariat général, 1920, avril, p. 90-96.

10. - VAN DEN DRIESSCHE, Bernard. Les moulages d'Athènes : 1920-1930. [Document électronique]. Louvain-la-Neuve: Musée de Louvain-la-Neuve, 2008, site: http://web.archive.org/web/*/ http://www.muse.ucl.ac.be/Castlisting/Castlisting0.html [consulté 07/03/2016].

11. - Archives administratives des Musées royaux d'art et d'histoire de Bruxelles. Atelier des moulages. Dossier 52, Commandes 1926-1928, documents 682.

12. - Suite aux travaux de restauration du bâtiment, la séance n'a pas pu coïncider avec l'année réelle de l'anniversaire (1425-1925) mais bien les 28 et 29 juin 1927. LADEUZE, Paulin Mgr. «Discours de la rentrée académique ». Annuaire de l'Université catholique de Louvain, 1927-1929, p. 325.

13. - MAYENCE, Fernand. Hommage de reconnaissance de l'Université de Louvain à la Grèce. Louvain, 1930, 6 pages. MAYENCE, Fernand. Université catholique de Louvain. Programme de la séance académique organisée aux halles universitaires le jeudi 22 mai 1930 à l'occasion de l'inauguration de la statue de Thémis et de la collection de moulages d'œuvres d'art antique offerte à l'Université de Louvain par le gouvernement hellénique. Louvain, s.d. [1930], 8 pages. 
14. - Archives du musée de Louvain-la-Neuve. Fonds F. Mayence. Carnet de notes. Voyages 1928-1930. Lyon.

15. - La part restée propriété de la Katholieke Universiteit Leuven a fait l'objet d'un premier catalogue de 234 numéros en 1975. SCHLUSMANS, Frida. Kennismaking met de griekse en romeinse sculptuur. Leuven, 1975. PROVOOST, Arnold. De afgietselverzameling klassieke sculpturen aan de Katholieke Universiteit Leuven. Leuven, 2004. Elle a été entièrement réorganisée et inaugurée le 6 février 2015.

16. - HENNAUT, Éric et LIESENS, Liliane. Henry Lacoste architecte (1885-1968). Bruxelles : Archives d'architecture moderne, 2008, p. 154-160.

17. - Lettre du 27 août 1931 de Fernand Mayence à Frans Cumont, citée mais non publiée par BONNET, Corinne. La correspondance scientifique de Franz Cumont conservée à l'Academia Belgica de Rome. Bruxelles-Rome : Institut historique belge de Rome. Études de philologie, d'archéologie et d'histoire ancienne, 35,1997, p. 304.

18. - Un exemplaire est conservé au musée de Louvain-la-Neuve; l'autre l'est au Museo della Civiltà Romana à Rome (confirmation de Mme Clotilde d'Amato, conservatrice).

19. - «J'attends Lacoste qui doit arriver aujourd'hui ou demain; il a engagé à Rome un mouleur qui sera chargé de prendre des moulages des principales choses découvertes ; il est déjà arrivé hier m'apportant une lettre de Lacoste ». Archives du musée de Louvain-la-Neuve. Fonds F. Mayence. Fouilles d'Apamée/Correspondance. Lettre du 9 octobre 1931 de F. Mayence à Beyrouth, à son épouse. "Aujourd'hui nous avons fait le premier envoi de moulages à Beyrouth pour y être embarqués à destination de la Belgique. Vous savez, n'est-ce pas, que nous faisons exécuter ici par un mouleur italien des copies en plâtre des principaux fragments d'architecture et de sculpture que nous avons découverts ; grâce à ces moulages, il sera possible de reconstituer au Musée quelques travées de la célèbre colonnade d'Apamée. Si notre plan réussit, je crois que ce sera très intéressant ». Id. Lettre du mardi 17 novembre 1931 de F. Mayence à Kalaât-elMoudik, à son épouse.

20. - « Le mouleur italien dont nous sommes extrêmement satisfait, travaille d'arrache-pied pour terminer son travail. Voici une photo qui le montre à côté de quelques tambours de colonne qu'il vient d'achever. Puissent toutes ces copies arriver en bon état à Bruxelles »! Id. Lettre du 27 novembre 1931 de F. Mayence à Kalaât-el-Moudik, à son épouse. Le bilan de cette campagne est évoqué dans une correspondance adressée à F. Cumont dans laquelle F. Mayence revient sur l'épineuse question du transport du « butin » qui représente « 15 tonnes de pierres et ca $24 \mathrm{~m}^{3} \mathrm{de}$ moulages. ». Lettre du 27 décembre 1932 de F. Mayence à Bruxelles à F. Cumont, citée mais non publiée par BONNET, Corinne. Op.cit., p. 308.

21. - Lettre du 13 octobre 1931 de F. Mayence à Frans Cumont. Id.

22. - MAYENCE, Fernand. «La salle d'Apamée ». Bulletin des Musées royaux d'art et d'histoire, 1933, n ○3 (mai), p. 50-57. PEETERS, Félix. "Le portique d'Apamée». Revue du Cercle des alumni de la Fondation universitaire, 1933, IV, $\mathrm{n}^{\circ} 4$ (avril), p. 3-5.

23. - Extrait d'une communication de Charles Leplae (sculpteur) faite à l'Académie Picard à Bruxelles le 6 décembre 1946 dénonçant la disparition du musée des moulages à la fin des années 1920. Archives administratives des MRAH. Atelier des moulages. Dossier 117/11. Six pages dactylographiées.

24. - RUYSSINCK, Micheline. «1946-1984». Dans Liber Memorialis - 1835-1985. MRAH-KMKG, Bruxelles, 1985, p. 57.

25. - PUTTEMANS, Pierre. Robert Puttemans architecte - Ou la passion de la mesure. Bruxelles : éd. Civa, 2012, p. 78 et 88.

26. - Voir récemment : La maquette. Un outil au service du projet architectural. Colloque international. 20-21 mai 2011 [document électronique]. Paris: Cité de l'architecture, 2011, site http:// www.citechaillot.fr/data/auditorium_c49f4/fiche/911/brochurecolloquemaquettes_2968a.pdf [consulté 07/03/2016]. 
27. - Parmi d'autres exemples: Archivé - Préserver la Grèce antique en 3D; Modèle 3D du portique des caryatides [document électronique]. Ottawa : ITI-CNRC, 2010, site http://www.nrc-cnrc.gc.ca/fra/ realisations/saillants/2010/grece_3d.html [consulté 07/03/2016].

28. - FLOUR, Isabelle. "Inventions et réinventions du musée de moulages d'architecture, Londres-Paris-Liverpool 1851-1887 ». Dans ROLLAND, Anne-Solène et MURAUSKAYE, Hanna (dir.). Les musées de la nation. Créations, transpositions, renouveaux. Europe, XIX ${ }^{e}-\mathrm{XXI} I^{e}$ siècles. Paris : l'Harmattan, 2008, p. 221-239.

\section{RÉSUMÉS}

Fernand Mayence (1879-1959) est une figure majeure de l'archéologie classique en Belgique. Attaché à l'université catholique de Louvain (UCL) à partir de 1907 et à la section des Antiquités des Musées royaux d'art et d'histoire de Bruxelles à partir de 1913, il y concrétisa respectivement le «musée d'Archéologie classique » en 1927 et la «salle d'Apamée sur l'Oronte » en 1933. Ces réalisations occupent une place importante dans l'histoire des collections de moulages en Belgique dans la première moitié du $\mathrm{xx}^{\mathrm{e}}$ siècle.

Fernand Mayence (1879-1959) was a major figure in classical archeology in Belgium. Attached to the Catholic University of Louvain (UCL) from 1907 and to the Antiquities section of the Royal Museums of Art and History in Brussels from 1913, he was the founder, successively, of the Museum of Classical Archaeology at Louvain in 1927 and of the Room of Apamea on the Orontes in 1933 in Brussels. These achievements are significant ones in the history of collections of plaster casts in Belgium during the first half of the twentieth century.

\section{INDEX}

Mots-clés : Fernand Mayence, art antique, archéologie, Musée des moulages, Université catholique de Louvain, Musées royaux d'art et d'histoire de Bruxelles, fouilles d'Apamée (Syrie), reconstitution architecturale

Keywords : Antiquity, Archaeology, Plaster cast museum, Catholic university of Louvain, Royal museums of art and history in Brussels, archaeological excavations at Apamée (Syria), architectural reconstitution 